\title{
Levantamento florístico das macroalgas da baía de Sepetiba e adjacências, RJ: ponto de partida para o Programa GloBallast no Brasil
}

\author{
Maria Teresa Menezes de Széchy ${ }^{1,5}$, Gilberto Menezes Amado Filho², Valéria Cassano ${ }^{3}$, Joel Campos De-Paula ${ }^{3}$, \\ Maria Beatriz de Barros Barreto ${ }^{4}$, Renata Perpétuo Reis ${ }^{2}$, Bianca Veras Marins-Rosa ${ }^{2}$ e Fátima Malheiro Moreira ${ }^{3}$
}

\author{
Recebido em 09/03/2004. Aceito em 04/02/2005
}

\begin{abstract}
RESUMO - (Levantamento florístico das macroalgas da baía de Sepetiba e adjacências, RJ: ponto de partida para o Programa GloBallast no Brasil). O Porto de Sepetiba, Estado do Rio de Janeiro, foi escolhido, no Brasil, como área-piloto do projeto "Remoção de barreiras para a implementação efetiva do controle de água de lastro e medidas de gestão em países em desenvolvimento" (Programa GloBallast). Para possibilitar comparações, os seis países participantes do Programa GloBallast foram orientados a adotar a mesma metodologia para o levantamento preliminar da biota das áreas-pilotos valorizando a identificação em nível de espécie. O presente estudo teve como objetivo aplicar metodologia estabelecida pelo Centro para Pesquisa sobre Espécies Marinhas Introduzidas, da Austrália (Procedimento CRIMP), com algumas adaptações, no levantamento florístico das macroalgas de substratos consolidados naturais da área-piloto do Porto de Sepetiba. Coletas de macroalgas foram realizadas em novembro/2001, em nove locais da baía de Sepetiba e adjacências, desde a franja da região sublitorânea até cerca de $-4 \mathrm{~m}$ de profundidade. Em cada local, seis quadrados de $0,1 \mathrm{~m}^{2}$ de área foram raspados. $\mathrm{O}$ material foi depositado no Herbário do Instituto de Pesquisas Jardim Botânico do Rio de Janeiro (RB). Das 96 espécies identificadas (20 Chlorophyta, 16 Phaeophyta, 60 Rhodophyta), 12 não haviam sido citadas anteriormente para a área de estudo. Cladophora pellucidoidea C. Hoek, Coelothrix irregularis (Harv.) Boergesen e Acrochaetium savianum (Menegh.) Nägeli são ocorrências novas para o Estado. Não há indícios de que as novas ocorrências equivalem a espécies recentemente introduzidas por meio das atividades portuárias. O procedimento metodológico adotado mostrou-se eficiente, resultando em listagem de espécies comparável às de levantamentos florísticos com maior esforço amostral e complementando os dados pretéritos existentes para a baía de Sepetiba e adjacências. No entanto, ressalta-se a importância de uma reavaliação das escalas temporal e espacial da estratégia de amostragem, em estudos futuros. Recomenda-se a padronização metodológica para o levantamento florístico de áreas portuárias como a baía de Sepetiba, e de outras áreas do litoral brasileiro sujeitas à interferência do homem, tomando por base o procedimento CRIMP.
\end{abstract}

Palavras-chave: água de lastro, baía de Sepetiba, florística, macroalgas, monitoramento

\begin{abstract}
Floristic survey of the macroalgae from Sepetiba bay and surrounding area, Rio de Janeiro State: starting point for the GloBallast Programme in Brazil). The Port of Sepetiba, State of Rio de Janeiro, was chosen as the Brazilian pilot area for the project "Global Ballast Water Management Programme" (GloBallast Programme). For comparative purposes, six countries were guided by the GloBallast Programme to follow the same methodology, emphasizing identification of organisms to the specific level during the preliminary biota survey. This study aimed to apply the methodology established by the Australian Centre for Research on Introduced Marine Pests (CRIMP procedures) for the floristic survey of macroalgae of natural hard substrate of the area of Port of Sepetiba. Macroalgal samplings were carried out in November 2001 at nine sites situated in Sepetiba Bay and adjacent area, from the sublittoral fringe to ca. - $4 \mathrm{~m}$ deep. At each site, six $0.1 \mathrm{~m}^{2}$ quadrates were scraped. Specimens were sent to the Herbarium of the "Instituto de Pesquisas Jardim Botânico do Rio de Janeiro" (RB). Ninety six species were identified (20 Chlorophyta, 16 Phaeophyta, and 60 Rhodophyta); 12 species have not been cited to the study area by the preceding studies. Cladophora pellucidoidea C. Hoek, Coelothrix irregularis (Harv.) Boergesen, and Acrochaetium savianum (Menegh.) Nägeli are recorded for the first time to the state of Rio de Janeiro. There are no evidences to conclude that the new records correspond to recently introduced species through the port activities. The methodological procedures proved to be efficient because they produced a species list to be comparable to those of taxonomic surveys with higher sampling effort. They also enlarged the existing information about the phycological flora of Sepetiba Bay. Nevertheless, for future studies, we point out the importance of a re-evaluation of the temporal and spatial scales of the sampling strategy. We recommend the methodological standardization of floristic surveys for port areas such as Sepetiba Bay, as well as other Brazilian coastal areas subjected to human interferences, taking into account the CRIMP procedures.
\end{abstract}

Key words : ballast water, Sepetiba bay, floristic survey, macroalgae, monitoring procedures

\footnotetext{
${ }^{1}$ Universidade Federal do Rio de Janeiro, Centro de Ciências da Saúde, Bloco A, Instituto de Biologia, Departamento de Botânica, Cidade Universitária, Ilha do Fundão, CEP 21941-590, Rio de Janeiro, RJ, Brasil

2 Instituto de Pesquisas Jardim Botânico do Rio de Janeiro, Programa Zona Costeira, Rua Pacheco Leão 915, Jardim Botânico, CEP 22460-030, Rio de Janeiro, RJ, Brasil

3 Universidade do Estado do Rio de Janeiro, Instituto de Biologia Roberto Alcântara Gomes, Departamento de Biologia Vegetal, Rua São Francisco Xavier 524, PHLC, sala 511, Maracanã, CEP 20550-013, Rio de Janeiro, RJ, Brasil

4 Universidade Federal Rural do Rio de Janeiro, Instituto de Biologia, Departamento de Botânica, Antiga Rodovia Rio-São Paulo km 47, Seropédia, CEP 23890-000, Rio de Janeiro, RJ, Brasil

5 Autor para correspondência: szechy@biologia.ufrj.br
} 


\section{Introdução}

Ao longo da História do Brasil, o transporte marítimo ocupou papel de destaque em diferentes momentos. No início, o lastro das embarcações era feito com material de origem terrestre, como pedras e areia. A partir da década de 1880 , passou a ser mais comum o uso da própria água do mar como lastro (Carlton 1985; 2001). Diversas outras melhorias tecnológicas, juntamente com a industrialização e a expansão do comércio intercontinental, têm resultado na crescente ampliação do transporte marítimo. Atualmente, espécies podem chegar a águas costeiras brasileiras de todas as regiões do planeta, viajando através de inúmeras rotas. O aumento de rotas no transporte marítimo, além do número e velocidade dos navios, tem levado ao incremento de problemas ambientais criados por espécies introduzidas, que podem ser transportadas pela água de lastro ou nos cascos dos navios (Carlton \& Geller 1993; Grosholz et al. 2000; Ruiz et al. 2000). Exemplos da introdução acidental de espécies de macroalgas, tendo como possível vetor os navios, vêm sendo relatados na literatura (Hay 1990; Verlaque 1994; VillalardBohnsack \& Harlin 1997; Carlton 2001; Smith et al. 2002). No entanto, poucos são os estudos que tratam de espécies de macroalgas introduzidas no litoral brasileiro, acidentalmente ou não (Oliveira 1984; Paula et al. 1998; Horta \& Oliveira 2000; Bellorín \& Oliveira 2001; Paula \& Oliveira 2004).

Em termos mundiais, a taxa de invasões por espécies no ambiente marinho está crescendo de modo alarmante (Cohen \& Carlton 1998; Carlton 2001; Boudouresque \& Verlaque 2002). Nesse contexto, a Organização Marítima Internacional (IMO) - agência das Nações Unidas responsável pela segurança da navegação e pela prevenção da poluição marinha por navios, juntamente com o Programa de Desenvolvimento das Nações Unidas (UNDP) e o Fundo Mundial para o Meio Ambiente (GEF), criaram o projeto "Remoção de Barreiras para a Implementação Efetiva do Controle de Água de Lastro e Medidas de Gestão em Países em Desenvolvimento", conhecido como Programa GloBallast, a fim de prover assistência técnica a esses países, no sentido de propiciar a elaboração e a implementação de estratégias de monitoramento da biodiversidade, específicas para cada porto e país. Dentro deste projeto, seis países foram escolhidos para participar através da execução de um estudo-piloto: África do Sul, Brasil, China, Índia, Irã e Ucrânia. Para possibilitar comparações futuras, todos os países participantes do Programa GloBallast foram orientados a adotar o mesmo procedimento metodológico no levantamento preliminar da biota das áreaspilotos. Este procedimento, denominado de Procedimento CRIMP, foi estabelecido pelo Centro de Pesquisa Sobre Espécies Marinhas Introduzidas da Austrália (CSIRO).

No Brasil, foi definido o Porto de Sepetiba, no Estado do Rio de Janeiro, como área-piloto, tendo sido o projeto-piloto coordenado pelo Ministério do Meio Ambiente (Leal Neto \& Jablonski 2004).

A flora ficológica da baía de Sepetiba foi extensivamente estudada por A.G. Pedrini (dados não publicados) que, através de coletas na década de 1970, em 79 localidades com diferentes ambientes, citou 171 espécies de macroalgas (34 Chlorophyta, 26 Phaeophyta e 111 Rhodophyta). Mais recentemente, citações de macroalgas para a Baía de Sepetiba foram feitas por Reis \& Yoneshigue-Valentin (1998), Széchy \& Paula (2000), Tavares (2001) e Amado Filho et al. (2003). Amado Filho et al. (2003) caracterizaram a estrutura de comunidades fitobentônicas da região sublitorânea das Ilhas Duas Irmãs, do Gato, do Martins e Itacuruçá e da Ponta da Marambaia, listando 92 espécies (18 Chlorophyta, 14 Phaeophyta e 60 Rhodophyta), sendo 16 novas citações para a área. Portanto, os dados pretéritos apontam para uma rica flora ficológica na Baía de Sepetiba, em um passado recente (Amado Filho \& Marins 2004).

O Procedimento CRIMP - Determina que o levantamento preliminar da biota de cada área-piloto deve fornecer: i) o levantamento das espécies nativas; ii) o levantamento das espécies introduzidas e criptogênicas; iii) a distribuição e a abundância relativa de um número limitado de espécies, indicadas como potencialmente invasoras. Para tanto, define quatro pré-requisitos: 1) desenho amostral com um mínimo de padronização; 2) identificação do material biológico em nível de espécie, sempre que possível; 3) identificação das espécies verificada por especialistas; 4) coleção de referência depositada em instituição científica reconhecida internacionalmente.

Ressalta a importância de compatibilizar as estratégias amostrais, necessárias a atender aos prérequisitos e objetivos acima discriminados, com questões de ordem prática, como a disponibilidade de recursos financeiros e de tempo.

Os métodos de amostragem devem ser selecionados para garantir o estudo de hábitats diferentes, como massa d'água, substratos não consolidados e consolidados, naturais e artificiais, e 
para fornecer dados qualitativos (presença ou ausência) ou quantitativos (abundância). A amostragem deve ser estratificada (por hábitats), com réplicas, e preferencialmente aleatória. $\mathrm{O}$ desenho amostral deve possibilitar a detecção de espécies "raras", com distribuição e abundância limitadas. Análise usando o método de Green \& Young (1993, apud Hewitt \& Martin 2001), feita para determinar o esforço amostral apropriado para espécies raras com distribuição de Poisson, sugere que o tamanho amostral de aproximadamente 20 unidades amostrais (quadrado) é necessário para detectar uma espécie com densidade média de 0,1 indivíduo por quadrado.

Assim, para substratos consolidados são recomendadas três unidades amostrais em pelo menos sete locais, selecionados de acordo com sua prioridade. Locais com prioridade máxima para o levantamento são aqueles mais próximos das instalações do porto ou mais influenciados pelo despejo de água de lastro, como construções e equipamentos diretamente relacionados às atividades de navegação comercial (rampas e pilares de atracadouros em uso, atracadouros inativados, sinalizadores e bóias) ou às atividades de pesca e de recreação próximas ao porto.

Em locais onde existem substratos consolidados em profundidades maiores (-7 m ou mais), é indicada a realização de três transectos de linha, dispostos verticalmente a partir do nível médio das marés baixas, e distantes entre si $10 \mathrm{~m}$ a $15 \mathrm{~m}$. Tais transectos devem ser fotografados ou filmados; ao longo dos mesmos, a $-0,5 \mathrm{~m},-3,0 \mathrm{~m}$ e -7,0 $\mathrm{m}$ de profundidade, devem ser lançados três quadrados de $0,10 \mathrm{~m}^{2}$. Em locais mais rasos, com substratos consolidados presentes apenas até $-5 \mathrm{~m}$ de profundidade, é indicada a realização de dois transectos de linha, dispostos horizontalmente a $-0,5 \mathrm{~m}$ de profundidade e no fundo, ao longo dos quais devem ser lançados aleatoriamente cinco quadrados de $0,10 \mathrm{~m}^{2}$. A fauna e a flora fixadas no interior desses quadrados devem ser cuidadosamente raspadas. As macroalgas triadas devem ser logo herborizadas ou mantidas em solução tamponada de formalina a $7 \%$.

Amostragens na região entre marés são indicadas como opcionais pelo procedimento CRIMP. Segundo Hewitt \& Martin (2001), espécies introduzidas têm maior probabilidade de se estabelecerem na região sublitorânea, quando comparada com a região entre marés.

O presente estudo teve como objetivo aplicar o procedimento CRIMP no levantamento florístico das macroalgas de substratos consolidados naturais da área-piloto do Porto de Sepetiba, como ponto de partida do Programa GloBallast no Brasil (Projeto IMO/ PNUD/GEF GLO/99/G31/A/1G/19).

\section{Material e métodos}

A área de estudo, que abrangeu a Baía de Sepetiba e o lado da Ilha Grande voltado para a saída da Baía de Sepetiba, foi dividida em quatro subáreas, de acordo com sua prioridade para o Programa GloBallast. A subárea de prioridade máxima (prioridade um, segundo o procedimento CRIMP) foi definida como a de maior chance de acolher espécies introduzidas por água de lastro, especialmente invertebrados, englobando os pilares do Porto de Sepetiba, as bóias de sinalização do canal de entrada das embarcações e alguns piers de localidades próximas ao porto. Por outro lado, a subárea de prioridade mínima (prioridade quatro) foi definida como a de menor possibilidade de acolher espécies introduzidas por água de lastro, incluindo os costões rochosos da Ilha Grande e proximidades (Fig. 1).

O levantamento florístico baseou-se em coletas realizadas na primavera de 2001 , em nove locais (Fig. 1), classificados como de prioridades dois ou quatro (Tab. 1). As subáreas de prioridades um e três não foram consideradas neste estudo por apresentarem apenas substratos consolidados artificiais, em sua maioria posicionados na vertical e em locais sombreados, com densa cobertura de invertebrados sésseis. As coordenadas dos locais de coleta foram registradas com GPS (Garmin, modelo $12 \mathrm{XL}$ ).

Em cada local, quadrados de $0,10 \mathrm{~m}^{2}$ de área interna foram posicionados ao acaso sobre as rochas, desde a franja da região sublitorânea até cerca de $-4 \mathrm{~m}$ de profundidade ( $n=6$ ou 5), perfazendo o total de 53 unidades amostrais, a maioria em locais de prioridade 2. Em alguns locais, onde a comunidade bentônica epilítica da região sublitorânea rasa mostrou-se homogênea quanto à cobertura de macroalgas, as unidades amostrais foram lançadas em apenas um nível (nível superior: da franja da região sublitorânea até cerca de $-2 \mathrm{~m}$ de profundidade). Em outros locais, com substrato rochoso natural ocorrendo mais no fundo e onde a comunidade bentônica mostrou-se diferenciada em mais de uma zona quanto à cobertura de macroalgas, as unidades amostrais foram lançadas em dois níveis (nível superior e nível inferior: entre -3 m e $-4 \mathrm{~m}$ de profundidade) (Tab. 1). Todos os organismos fixados na área interna das unidades amostrais foram retirados com auxílio de espátulas metálicas. As profundidades das coletas foram registradas com profundímetro no momento das raspagens. As coletas 


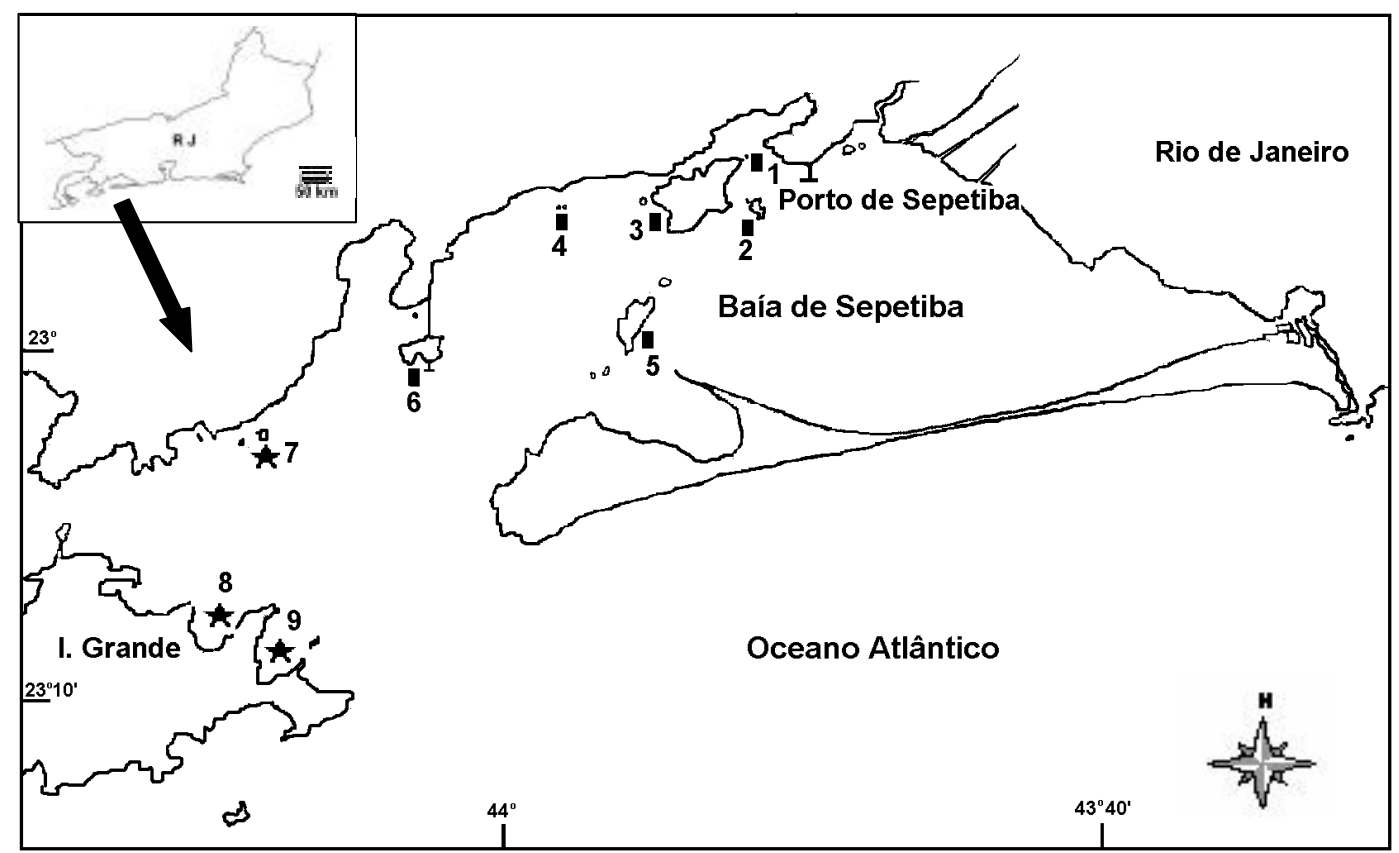

Figura 1. Locais de coleta de macroalgas na baía de Sepetiba e adjacências, RJ, Brasil, de acordo com sua prioridade para o Programa GloBallast. 1 - Ilha do Gato; 2 - Ilha do Martins; 3 - Praia Grande, Ilha de Itacuruçá; 4 - Ilha Duas Irmãs; 5 - Praia do Catita, Ilha de Jaguanum; 6 - Ilha Guaíba; 7 - Ilha de Cotiataçu; 8 - Enseada de Abraão; 9 - Enseada de Palmas. $\mathbf{\square}=$ prioridade 2; $\star$ = prioridade 4, segundo o procedimento CRIMP.

foram realizadas através de mergulho autônomo.

Macroalgas foram separadas dos representantes do zoobentos e fixadas em solução de formaldeído em água do mar a $4 \%$. Estas amostras de macroalgas passaram por triagem mais minuciosa, sob microscópio estereoscópico, sendo aproveitados também os espécimes de menores dimensões, epífitas ou emaranhados nos tufos das macroalgas.

Foram seguidas a classificação taxonômica e a nomenclatura adotada por Wynne (1998). Para alguns gêneros, houve a complementação através de outros estudos taxonômicos: Cladophora (Silva et al. 1996), Jania e Titanoderma (Chamberlain \& Irvine 1994; Moura \& Yamaguishi-Tomita 1998) e Polysiphonia

Tabela 1. Coletas de macroalgas realizadas em substratos consolidados naturais da região sublitorânea rasa da Baía de Sepetiba e adjacências, RJ, Brasil: locais, coordenadas, datas, níveis do costão, e classificação quanto à prioridade para o levantamento de espécies introduzidas, segundo o Procedimento CRIMP: 2 - prioridade intermediária, com razoável possibilidade de acolher espécies introduzidas, devido à pouca distância para os locais de despejo de água de lastro; 4 - prioridade mínima, com a menor possibilidade de acolher espécies introduzidas.

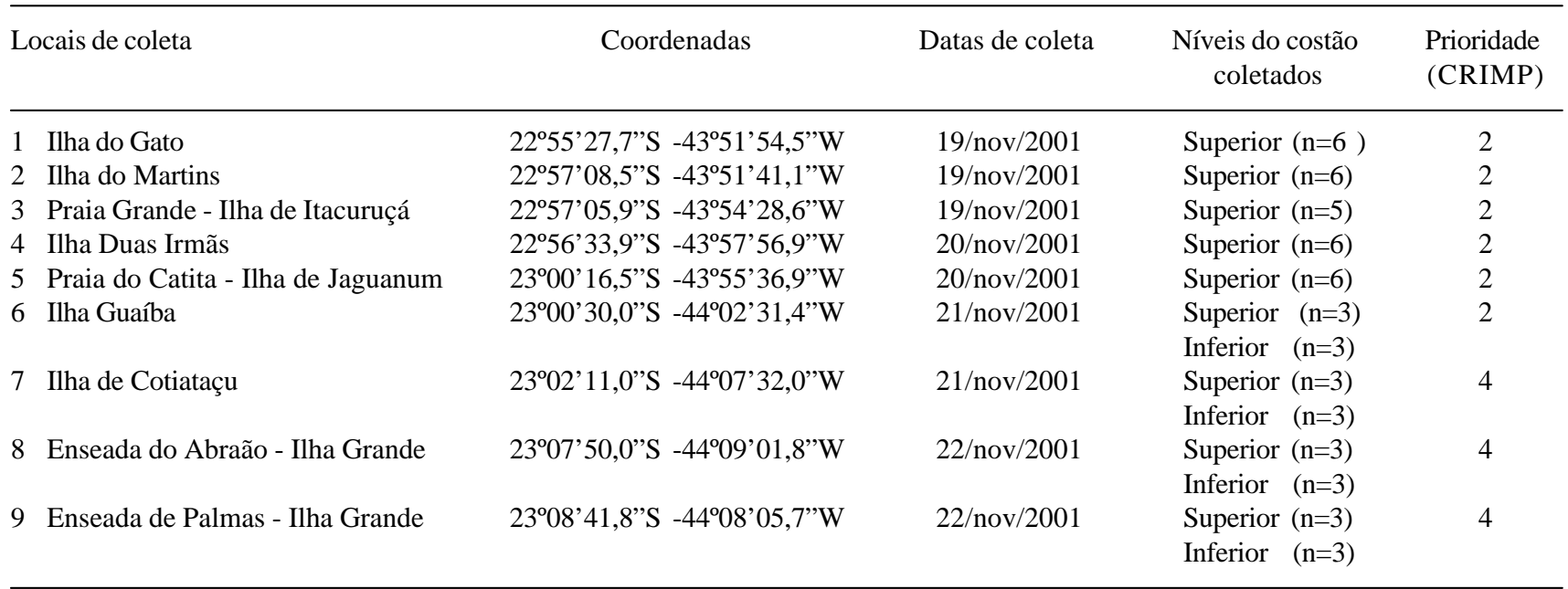


(Guimarães et al. 2004). Para determinados táxons, houve confirmação da identificação por especialistas (Tab. 2).

O material foi depositado no Herbário do Instituto de Pesquisas Jardim Botânico do Rio de Janeiro (RB), tanto herborizado como em meio líquido, no caso das espécies mais delicadas.

\section{Resultados e discussão}

Foram identificadas 96 espécies de macroalgas, sendo 20 Chlorophyta, 16 Phaeophyta e 60 Rhodophyta (Tab. 2). A importância da participação de especialistas em grupos diferentes foi indiscutível para o aprimoramento taxonômico da listagem de espécies

Tabela 2. Macroalgas de comunidades bentônicas de substratos naturais de nove locais na baía de Sepetiba e adjacências, RJ, Brasil. 1 - Ilha do Gato; 2 - Ilha do Martins; 3 - Praia Grande, Ilha de Itacuruçá; 4 - Ilha Duas Irmãs; 5- Praia do Catita, Ilha de Jaguanum; 6 - Ilha Guaíba; 7 - Ilha de Cotiataçu; 8- Enseada de Abraão, Ilha Grande; 9- Enseada de Palmas, Ilha Grande. X= presente; RB = presente, depositado no Herbário do Instituto de Pesquisas Jardim Botânico do Rio de Janeiro. * = material identificado por especialista: ${ }^{1}=$ Moura, C.W.N.; ${ }^{2}=$ Fujii, M.T.

\begin{tabular}{|c|c|c|c|c|c|c|c|c|c|}
\hline \multirow[b]{2}{*}{ Táxons } & \multicolumn{9}{|c|}{ Locais } \\
\hline & 1 & 2 & 3 & 4 & 5 & 6 & 7 & 8 & 9 \\
\hline \multicolumn{10}{|l|}{ CHLOROPHYTA } \\
\hline 1. Enteromorpha compressa (L.) Nees & - & - & $\mathrm{RB}$ & - & - & $\mathrm{X}$ & - & - & - \\
\hline 2. E. flexuosa (Wulfen) J. Agardh subsp.flexuosa & & $\mathrm{RB}$ & $\mathrm{RB}$ & $\mathrm{X}$ & $\mathrm{X}$ & $\mathrm{RB}$ & $\mathrm{RB}$ & - & $\mathrm{RB}$ \\
\hline 3. Ulva fasciata Delile & $\mathrm{X}$ & $\mathrm{RB}$ & - & - & $\mathrm{X}$ & $\mathrm{RB}$ & - & - & $\mathrm{X}$ \\
\hline 4. $U$. rigida $\mathrm{C}$. Agardh & - & - & $\mathrm{X}$ & - & - & $\mathrm{RB}$ & $\mathrm{RB}$ & - & - \\
\hline 5. Entocladia viridis Reinke & - & - & $\mathrm{RB}$ & - & - & - & $\mathrm{X}$ & - & - \\
\hline 6. Chaetomorpha aerea (Dillwyn) Kütz. & - & - & - & - & - & - & $\mathrm{X}$ & - & $\mathrm{RB}$ \\
\hline 7. C. brachygona Harv. & - & - & $\mathrm{RB}$ & - & - & $\mathrm{X}$ & - & - & - \\
\hline 8. Cladophora brasiliana $\mathrm{G}$. Martens & - & - & - & - & - & - & $\mathrm{X}$ & $\mathrm{RB}$ & $\mathrm{X}$ \\
\hline 9. C. capensis (C. Agardh) De Toni & - & $\mathrm{X}$ & $\mathrm{X}$ & $\mathrm{RB}$ & $\mathrm{X}$ & $\mathrm{RB}$ & $\mathrm{RB}$ & - & $\mathrm{RB}$ \\
\hline 10. C. coelothrix Kütz. & - & - & - & - & $\mathrm{RB}$ & $\mathrm{RB}$ & - & - & - \\
\hline 11. C. pellucidoidea C. Hoek & - & - & - & - & - & - & - & - & $\mathrm{RB}$ \\
\hline 12. C. vagabunda (L.) C. Hoek & - & - & $\mathrm{RB}$ & - & $\mathrm{RB}$ & $\mathrm{X}$ & - & $\mathrm{X}$ & $\mathrm{RB}$ \\
\hline 13. Rhizoclonium riparum (Roth) Kütz. ex Harv. & - & $\mathrm{X}$ & - & - & $\mathrm{RB}$ & - & - & - & - \\
\hline 14. Cladophoropsis membranacea (C. Agardh) Boergesen & - & - & - & - & $\mathrm{RB}$ & - & - & - & - \\
\hline 15. Bryopsis pennata J.V. Lamour. & $\mathrm{RB}$ & - & $\mathrm{X}$ & - & - & $\mathrm{X}$ & $\mathrm{RB}$ & - & - \\
\hline 16. B. plumosa (Huds.) C. Agardh & - & $\mathrm{RB}$ & - & - & - & $\mathrm{RB}$ & - & - & - \\
\hline 17. Derbesia tenuissima (Moris \& De Not.) P. Crouan \& H. Crouan & - & $\mathrm{RB}$ & - & - & - & $\mathrm{RB}$ & - & - & - \\
\hline 18. Codium decorticatum (Woodw.) M. Howe & - & $\mathrm{RB}$ & $\mathrm{RB}$ & - & $\mathrm{RB}$ & - & - & - & - \\
\hline 19. C. intertextum Collins \& Herv. & - & - & - & - & - & $\mathrm{X}$ & - & - & $\mathrm{RB}$ \\
\hline 20. C. taylorii P.C. Silva & - & $\mathrm{X}$ & - & - & $\mathrm{RB}$ & - & - & - & - \\
\hline \multicolumn{10}{|l|}{ PHAEOPHYTA } \\
\hline 1. Bachelotia antillarum (Grunow) Gerloff & - & - & - & - & - & - & $\mathrm{X}$ & - & - \\
\hline 2. Feldmannia indica (Sond.) Womersley \& A. Bailey & - & - & - & - & - & $\mathrm{X}$ & - & - & - \\
\hline 3. F. irregularis (Kütz.) Hamel & - & - & $\mathrm{X}$ & - & - & - & - & - & $\mathrm{X}$ \\
\hline 4. Hincksia mitchelliae (Harv.) P.C. Silva & - & $\mathrm{RB}$ & - & - & - & $\mathrm{RB}$ & - & - & - \\
\hline 5. Colpomenia sinuosa (Roth) Derbès \& Solier & - & - & $\mathrm{X}$ & - & - & $\mathrm{RB}$ & $\mathrm{RB}$ & - & $\mathrm{RB}$ \\
\hline 6. Sphacelaria brachygonia Mont. & - & - & - & - & - & - & - & $\mathrm{X}$ & $\mathrm{X}$ \\
\hline 7. S. rigidula Kütz. & - & - & - & - & - & - & $\mathrm{X}$ & $\mathrm{X}$ & - \\
\hline 8. S. tribuloides Menegh. & - & - & - & - & - & - & $\mathrm{RB}$ & $\mathrm{X}$ & $\mathrm{RB}$ \\
\hline 9. Sphacelaria spp. & - & - & $\mathrm{X}$ & - & - & $\mathrm{X}$ & - & - & - \\
\hline 9. Dictyopteris delicatula J.V. Lamour. & - & - & - & - & - & $\mathrm{RB}$ & $\mathrm{X}$ & - & - \\
\hline 10. Dictyota cervicornis Kütz. & - & - & - & $\mathrm{RB}$ & - & - & - & - & $\mathrm{RB}$ \\
\hline 11. Lobophora variegata (J.V. Lamour.) Womersley ex E.C. Oliveira & - & - & - & - & - & - & - & - & $\mathrm{RB}$ \\
\hline 12. Padina gymnospora (Kütz.) Sond. & $\mathrm{RB}$ & - & $\mathrm{X}$ & $\mathrm{RB}$ & - & - & - & $\mathrm{RB}$ & $\mathrm{RB}$ \\
\hline 13. Sargassum filipendula C. Agardh & - & $\mathrm{RB}$ & $\mathrm{RB}$ & - & - & - & - & - & - \\
\hline 14. S. furcatum Kütz. & - & - & - & - & - & - & - & - & $\mathrm{RB}$ \\
\hline 15. S. stenophyllum Mart. & - & $\mathrm{X}$ & $\mathrm{RB}$ & $\mathrm{RB}$ & - & - & - & - & - \\
\hline 16. S. vulgare C. Agardh & - & - & - & - & - & - & - & $\mathrm{RB}$ & - \\
\hline
\end{tabular}


Tabela 2 (continuação)

\begin{tabular}{llllllllll}
\hline & \multicolumn{3}{c}{ Locais } \\
\cline { 3 - 6 } Táxons & 1 & 2 & 3 & 4 & 5 & 6 & 7 & 8 & 9 \\
\hline
\end{tabular}

\section{RHODOPHYTA}

1. Stylonema alsidii (Zanardini) K.M. Drew

2. Erythrotrichia carnea (Dillwyn) J. Agardh

3. Sahlingia subintegra (Rosenv.) Kornmann

4. Acrochaetium microscopicum (Nägeli ex Kütz.) Nägeli

5. A. savianum (Menegh.) Nägeli

6. Jania adhaerens J.V. Lamour. ${ }^{* 1}$

7. J. cf. crassa J.V. Lamour. ${ }^{* 1}$

8. J. ungulata (Yendo) Yendo*1

9. Lithophyllum incrustans Philippi" ${ }^{* 1}$

10. Lithophyllum sp. $^{* 1}$

11. Titanoderma sp. $^{* 1}$

12. Amphiroa anastomosans Weber Bosse ${ }^{* 1}$

13. A. beauvoisii J.V. Lamour. ${ }^{* 1}$

14. A. fragilissima (L.) J.V. Lamour.

15. Gelidium crinale (Turner) Gaillon

16. G. pusillum (Stackh.) Le Jolis var. pusillum

17. Parviphycus tenuissimus (Feldmann \& Hamel) Santel. ${ }^{* 2}$

18. Asparagopsis taxiformis (Delile) Trevis.

19. Chondracanthus acicularis (Roth) Fredericq

20. C. teedei (Mertens ex Roth) Fredericq

21. Hypnea musciformis (Wulfen in Jacqu.) J.V. Lamour.

22. H. spinella (C. Agardh) Kütz.

23. Gymnogongrus griffithsiae (Turner) Mart.

24. Champia parvula (C. Agardh) Harv.

25. C. vieillardii Kütz.

26. Gastroclonium parvum (Hollenb.) C.F. Chang \& B.M. Xia

27. Lomentaria corallicola Boergesen

28. Coelothrix irregularis (Harv.) Boergesen

29. Gelidiopsis planicaulis (W.R. Taylor) W.R. Taylor

30. G. variabilis (Grev. ex J. Agardh) F. Schmitz

31. Aglaothamnion boergesenii (Aponte \& D.L. Ballant.)

L'Hardy-Halos \& Rueness in Aponte, D.L. Ballant. \& J.N. Norris

32. A. felliponei (M. Howe) Aponte, D.L. Ballant. \& J.N. Norris

33. A. uruguayense (W.R. Taylor) Aponte, D.L. Ballant. \& J.N. Norris

34. Anothrichium tenue (C. Agardh) Nägeli

35. Centroceras clavulatum (C. Agardh in Kunth) Mont. in Durieu de Maisonneuve

36. Centrocerocolax ubatubensis A.B. Joly

37. Ceramium brevizonatum H.E. Petersen var. caraibicum H.E. Petersen \& Boergesen

38. C. codii (H. Richards) Maz.

39. C. comptum Boergesen

40. C. deslongchampii Chauv. ex Duby

41. C. cf. diaphanum (Lightf.) Roth

42. C. flaccidum (Kütz.) Ardiss.

43. C. luetzelburgii O.C. Schmidt

44. C. tenerrimum (G. Martens) Okamura

45. C. vagans P.C. Silva

46. Ceramium clarionense Setchell \& Gardner

47. Spermothamnion nonatoi A.B. Joly

48. Spyridia filamentosa (Wulfen) Harv. in Hook.

49. Dasya brasiliensis E.C. Oliveira \& Y. Braga

50. Acanthophora spicifera (Vahl) Boergesen

51. Chondria cf. polyhriza Collins \& Herv. ${ }^{* 2}$

52. Herposiphonia secunda (C. Agardh) Ambronn

\begin{tabular}{|c|c|c|c|c|c|c|c|c|}
\hline X & - & $X$ & - & - & $\mathrm{RB}$ & $X$ & $X$ & $X$ \\
\hline- & - & $\mathrm{RB}$ & - & $X$ & $X$ & $X$ & - & $X$ \\
\hline- & - & $\mathrm{RB}$ & - & - & - & $X$ & $X$ & $X$ \\
\hline- & - & $\mathrm{RB}$ & - & - & $X$ & $X$ & - & $X$ \\
\hline- & - & - & - & - & - & $\mathrm{RB}$ & - & - \\
\hline- & $X$ & $X$ & $\mathrm{RB}$ & $X$ & $X$ & $\mathrm{RB}$ & $X$ & $\mathrm{RB}$ \\
\hline- & - & - & - & - & - & - & - & $\mathrm{RB}$ \\
\hline- & - & $X$ & - & - & - & $\mathrm{RB}$ & - & - \\
\hline- & - & - & - & - & - & - & - & $\mathrm{RB}$ \\
\hline- & - & - & - & - & - & $\mathrm{RB}$ & - & - \\
\hline- & - & $X$ & - & - & $\mathrm{RB}$ & $X$ & $X$ & $X$ \\
\hline- & - & - & - & - & - & - & - & $\mathrm{RB}$ \\
\hline- & - & $X$ & $\mathrm{RB}$ & - & $\mathrm{RB}$ & $\mathrm{RB}$ & $X$ & $X$ \\
\hline - & - & $X$ & - & - & $\mathrm{X}$ & $\mathrm{RB}$ & - & $X$ \\
\hline$X$ & - & $X$ & $\mathrm{RB}$ & $\mathrm{RB}$ & - & - & - & $X$ \\
\hline $\mathrm{X}$ & $\mathrm{RB}$ & - & $\mathrm{RB}$ & $\mathrm{RB}$ & $X$ & $X$ & $X$ & - \\
\hline- & - & - & - & - & - & - & - & $\mathrm{RB}$ \\
\hline- & - & - & - & - & $\mathrm{RB}$ & $X$ & - & $X$ \\
\hline $\mathrm{X}$ & $\mathrm{RB}$ & $\mathrm{RB}$ & $\mathrm{RB}$ & $\mathrm{RB}$ & $\mathrm{X}$ & $X$ & - & $X$ \\
\hline X & $\mathrm{RB}$ & $X$ & $\mathrm{RB}$ & $\mathrm{RB}$ & $X$ & $X$ & - & - \\
\hline- & $X$ & $\mathrm{RB}$ & $\mathrm{RB}$ & - & - & - & - & - \\
\hline$X$ & $X$ & $X$ & $\mathrm{RB}$ & $X$ & $\mathrm{RB}$ & $\mathrm{RB}$ & $X$ & $X$ \\
\hline$X$ & $\mathrm{RB}$ & $\mathrm{RB}$ & - & $\mathrm{RB}$ & - & - & - & - \\
\hline- & $\mathrm{RB}$ & - & $\mathrm{RB}$ & - & $\mathrm{RB}$ & $X$ & - & $\mathrm{RB}$ \\
\hline- & - & - & $\mathrm{RB}$ & - & $\mathrm{RB}$ & $\mathrm{RB}$ & $\mathrm{RB}$ & $X$ \\
\hline- & - & - & - & - & $\mathrm{RB}$ & - & - & \\
\hline- & - & $X$ & - & - & $\mathrm{RB}$ & $X$ & $X$ & $X$ \\
\hline- & - & - & - & - & $\mathrm{RB}$ & - & - & - \\
\hline$X$ & $\mathrm{RB}$ & $\mathrm{RB}$ & $\mathrm{RB}$ & $\mathrm{RB}$ & $\mathrm{RB}$ & $X$ & - & $X$ \\
\hline- & - & $\mathrm{RB}$ & $\mathrm{RB}$ & - & $\mathrm{X}$ & $X$ & $X$ & $X$ \\
\hline- & $\mathrm{RB}$ & - & - & $\mathrm{RB}$ & - & - & - & - \\
\hline- & $X$ & - & - & $\mathrm{RB}$ & - & - & - & - \\
\hline- & $\mathrm{RB}$ & $\mathrm{RB}$ & - & - & $X$ & $X$ & - & - \\
\hline $\mathrm{RB}$ & $\mathrm{X}$ & - & $X$ & $\mathrm{X}$ & - & - & - & - \\
\hline $\mathrm{RB}$ & $X$ & $\mathrm{RB}$ & $X$ & $X$ & $X$ & $X$ & $X$ & $X$ \\
\hline- & - & $\mathrm{RB}$ & - & - & - & - & - & - \\
\hline $\mathrm{X}$ & $X$ & $\mathrm{X}$ & $\mathrm{RB}$ & - & $X$ & - & $X$ & $X$ \\
\hline- & - & $X$ & - & - & $\mathrm{RB}$ & $\mathrm{RB}$ & - & - \\
\hline- & - & - & - & - & $\mathrm{X}$ & $\mathrm{RB}$ & $\mathrm{RB}$ & - \\
\hline- & - & - & - & - & $\mathrm{RB}$ & - & - & - \\
\hline- & - & - & - & - & - & $\mathrm{RB}$ & - & $\mathrm{RB}$ \\
\hline- & $\mathrm{RB}$ & $\mathrm{RB}$ & $X$ & - & $X$ & $X$ & $X$ & - \\
\hline- & - & - & - & - & $\mathrm{RB}$ & - & - & - \\
\hline- & - & $\mathrm{X}$ & - & - & $\mathrm{RB}$ & - & $X$ & - \\
\hline- & - & - & - & - & - & $X$ & - & $\mathrm{RB}$ \\
\hline$X$ & $\mathrm{RB}$ & $\mathrm{RB}$ & - & - & $X$ & $\mathrm{RB}$ & - & - \\
\hline- & - & $\mathrm{RB}$ & - & - & $X$ & X & - & $X$ \\
\hline$X$ & $\mathrm{RB}$ & $\mathrm{RB}$ & $\mathrm{RB}$ & $X$ & - & - & - & - \\
\hline- & - & $X$ & - & - & - & - & $\mathrm{RB}$ & - \\
\hline $\mathrm{X}$ & - & $\mathrm{RB}$ & $\mathrm{RB}$ & $\mathrm{X}$ & - & - & - & - \\
\hline- & - & - & - & - & - & - & - & $\mathrm{RB}$ \\
\hline- & - & - & - & - & $\mathrm{RB}$ & - & $X$ & $\mathrm{RB}$ \\
\hline
\end{tabular}


Tabela 2 (continuação)

\begin{tabular}{|c|c|c|c|c|c|c|c|c|c|}
\hline \multirow[b]{2}{*}{ Táxons } & \multicolumn{9}{|c|}{ Locais } \\
\hline & 1 & 2 & 3 & 4 & 5 & 6 & 7 & 8 & 9 \\
\hline 53. Laurencia intricata J.V. Lamour. ${ }^{* 2}$ & - & - & - & - & - & $\mathrm{RB}$ & - & - & - \\
\hline 54. Neosiphonia ferulacea (Suhr ex J. Agardh) S.M. Guim. \& M.T. Fujii & - & $\mathrm{RB}$ & - & - & $\mathrm{RB}$ & $\mathrm{X}$ & $\mathrm{X}$ & - & $\mathrm{RB}$ \\
\hline 55. N. tepida (Hollenb.) S.M. Guim. \& M.T. Fujii & - & - & $\mathrm{RB}$ & - & - & - & - & - & - \\
\hline 56. Polysiphonia denudata (Dillwyn) Grev. ex Harv. in Hook. & $\mathrm{RB}$ & $\mathrm{X}$ & - & $\mathrm{X}$ & - & $\mathrm{RB}$ & $\mathrm{X}$ & & $\mathrm{X}$ \\
\hline 57. P. howei Hollenb. in W.R. Taylor & - & - & - & - & - & $\mathrm{RB}$ & - & $\mathrm{X}$ & $\mathrm{X}$ \\
\hline 58. P. scopulorum Harv. & - & - & - & - & - & $\mathrm{RB}$ & - & - & - \\
\hline 59. P. subtilissima Mont. & - & - & $\mathrm{RB}$ & - & - & $\mathrm{X}$ & $\mathrm{RB}$ & $\mathrm{X}$ & $\mathrm{RB}$ \\
\hline 60. P. tongatensis Harv. ex Kütz. & $\mathrm{RB}$ & $X$ & - & $\mathrm{X}$ & $\mathrm{RB}$ & - & - & - & - \\
\hline
\end{tabular}

Números de registro dos materiais depositados no Herbário do Instituto de Pesquisas Jardim Botânico do Rio de Janeiro (RB): 376836 - 376846 ; 378210 - 378214; 378217 - 378233; 387871 - 387897; 390087 - 390143; 390320 - 390360; 390364 - 390368; 390384; 390388 - 390392; 391629 - 391633; 394915 - 394917. Sphacelaria spp. - material sem propágulos; Jania cf. crassa - material fragmentado; Lithophyllum sp. - pouca quantidade de material; concorda com a circunscrição do gênero fornecida por Chamberlain \& Irvine (1994); Titanoderma sp. - pouca quantidade de material; concorda com a circunscrição do gênero fornecida por Chamberlain \& Irvine (1994); Ceramium cf. diaphanum material não fértil; Chondria cf. polyhriza - pouca quantidade de material.

apresentada, atendendo a um dos pré-requisitos estabelecidos pelo procedimento CRIMP.

Cladophora pellucidoidea, Acrochaetium savianum e Coelothrix irregularis são ocorrências novas para o Estado do Rio de Janeiro. No Brasil, Cladophora pellucidoidea foi referida anteriormente apenas para o Estado da Paraíba, por A. Kanagawa (dados não publicados). Acrochaetium savianum foi citado antes, como A. sagreanum (Mont.) Bornet, para os Estados de São Paulo (Joly 1965) e Ceará (PinheiroVieira \& Ferreira 1968), enquanto Coelothrix irregularis apenas para o Estado de São Paulo (Joly 1965).

A ausência de material fértil dificultou a identificação de alguns táxons em nível específico, principalmente das ordens Corallinales e Ceramiales. Por exemplo, o material identificado como Ceramium cf. diaphanum mostrou similaridade com C. vagans, em relação à ramificação e à corticação nodal (Barreto \& Yoneshigue-Valentin 2001), mas se diferenciou deste por apresentar seis células periaxiais nas porções medianas do talo. O material analisado também se assemelhou a $C$. tenuissimum citado para o Estado do Paraná (Ugadim 1973, pr. 3, fig.1-5). A confirmação da identificação deste táxon seria possível apenas com a presença de tetrasporângios. Neste contexto, a coleção de referência depositada em instituição científica de renome é fundamental no sentido de dar oportunidade, no futuro, à reavaliação destes problemas taxonômicos, conforme recomenda o procedimento CRIMP.

Do total de espécies identificadas, 12 espécies correspondem a novas ocorrências para a baía de Sepetiba e Ilha Grande, a saber: Cladophora pellucidoidea, Derbesia tenuissima, Feldmannia indica, Acrochaetium savianum, Titanoderma sp., Jania ungulata, Amphiroa anastomosans, Parvyphycus tenuissimus, Lomentaria corallicola, Coelothrix irregularis, Aglaothamnion boergesenii e Laurencia intricata. Deste modo, o presente estudo complementou os dados pretéritos relativos à flora ficológica da baía de Sepetiba e adjacências. Algumas destas espécies (D. tenuissima, $F$. indica, C. irregularis, A. boergesenii e L. intricata) foram encontradas apenas em locais da baía de Sepetiba considerados com razoável possibilidade de acolher espécies introduzidas, devido a pouca distância para os locais de despejo de água de lastro (prioridade 2, intermediária). Dentre estes locais, destaca-se a Ilha Guaíba, que concentrou cerca de 58\% das ocorrências novas citadas neste estudo. Por outro lado, C. pellucidoidea, A. savianum, A. anastomosans e $P$. tenuissimus foram encontradas apenas em locais classificados como de prioridade mínima (4), ou seja, com a menor possibilidade de acolher espécies introduzidas por água de lastro. Não há indícios que possibilitem afirmar que as novas ocorrências equivalem a espécies recentemente introduzidas através das atividades portuárias. Pelo contrário, parece mais provável que muitas destas espécies, especialmente as de menores dimensões, não tenham sido coletadas ou triadas nos estudos anteriores ou tenham sido identificadas de modo incorreto, como $J$. ungulata e L. intricata.

A lista de macroalgas resultante da estratégia amostral usada neste estudo é comparável, em termos de número de espécies, à lista de Amado Filho et al. (2003), oriunda de coletas em substratos consolidados 
naturais da região sublitorânea rasa da baía de Sepetiba, em quatro épocas do ano de 1999, através de unidades amostrais de dimensões menores (quadrados de $0,04 \mathrm{~m}^{2}$ ), em cinco locais, sendo quatro deles também coletados no presente estudo. Nossos resultados também são comparáveis ao estudo das macroalgas da baía de Santos, litoral do Estado de São Paulo, na década de 1990 (Oliveira \& Qi 2003), o qual, através de coletas em 19 pontos durante dois anos, listou 91 espécies (22 Chlorophyceae, 11 Phaeophyceae, 58 Rhodophyceae). Esses autores ressaltam o considerável aumento do número de espécies em três dos pontos de coleta, quando comparado aos estudos de Oliveira Filho \& Berchez (1978) e de Berchez \& Oliveira (1992) na mesma área, levantando a hipótese da introdução de algumas espécies por água de lastro, sem, no entanto, especificar quais das 16 novas ocorrências podem ter sido introduzidas desta maneira. Por outro lado, comparando com os levantamentos florísticos realizados para a baía de Sepetiba, por A.G. Pedrini (dados não publicados), e para a baía da Ilha Grande, por Pedrini et al. (1994) e Figueiredo et al. (2004), o número de espécies do presente estudo foi expressivamente inferior, certamente em função das diferenças marcantes no esforço de coleta e na estratégia de amostragem. Os referidos estudos, essencialmente qualitativos, tiveram maior abrangência em termos de épocas, áreas e ambientes coletados.

Levando em consideração diferentes levantamentos florísticos realizados no litoral brasileiro, pode ser constatado que não existe uma padronização em relação ao esforço amostral, notando-se ampla variação quanto aos números de amostras e de pontos de coleta, ambientes, profundidades e épocas do ano. Neste contexto, ratifica-se a importância da proposta de padronização com base no procedimento CRIMP, para levantamentos preliminares da biota de áreas a serem monitoradas. Nossa experiência na área-piloto do Porto de Sepetiba nos faz concluir que o esforço amostral dispensado nas coletas (tamanho amostral de 53 unidades amostrais de $0,10 \mathrm{~m}^{2}$, em nove pontos de coleta e uma só época do ano) foi eficiente, resultando em listagem de espécies comparável em termos de número de espécies às de estudos com maior número de pontos de coleta, em diferentes épocas do ano.

As amostragens destrutivas realizadas foram imprescindíveis para o refinamento do tratamento taxonômico, especialmente de grupos reconhecidamente problemáticos, como Cladophora e Laurencia.
Amostragem destrutiva, juntamente com triagem minuciosa das amostras, garantiu a identificação de espécies pouco conspícuas, fixas ou emaranhadas em algas maiores, como Derbesia tenuissima e Coelothrix irregularis. Apesar de exigir maior esforço físico especialmente em locais de maior profundidade e correnteza, este tipo de coleta por raspagem de quadrados foi compatível com o tempo disponível para o trabalho no mar. Conforme recomenda Warwick (1993), ao se efetuar o planejamento de um novo estudo, o tempo e os custos requeridos para coleta e triagem de amostras devem ser considerados com cuidado.

No caso de estudos qualitativos prolongados, estratégias não destrutivas seriam as mais indicadas (De Wreede 1985), como "vídeo transect" e "visual searches", sugeridas pelo procedimento CRIMP (Hewitt \& Martin 2001). Além disso, amostragens estratificadas aleatórias seriam indispensáveis no caso de estudos comparativos, que visassem também a análise quantitativa das macroalgas (Krebs 1989; Underwood 1997), o que não foi objetivo do presente estudo.

Este trabalho constituiu, junto com os estudos de outros grupos de organismos, o ponto de partida para a implementação do Programa GloBallast no Brasil, favorecendo a avaliação da aplicação do procedimento CRIMP para o monitoramento da biota de áreas portuárias fora da costa da Austrália. Em relação às macroalgas, aponta-se algumas limitações da metodologia adotada neste estudo, que devem ser adequadas em futuros levantamentos florísticos, e também alguns pontos que podem ser aprimorados.

A principal limitação da metodologia adotada foi a restrição das coletas a substratos naturais. Acredita-se que substratos artificiais, em condições adequadas de luminosidade, possam abrigar espécies diferentes das encontradas neste estudo, concordando com os dados de A.G. Pedrini (dados não publicados), que verificou espécies de macroalgas sobre diversos tipos de substratos artificiais na baía de Sepetiba.

Um dos pontos da metodologia que merece ser aprimorado é a representatividade da amostragem em escala temporal e espacial. É recomendável a ampliação das coletas em termos de freqüência e duração, assim como em termos de extensão vertical no costão, de modo a melhor descrever a biodiversidade local. Dependendo das características topográficas e oceanográficas da área a ser estudada, a extensão vertical das amostragens deve incluir tanto a região entre marés como profundidades maiores da região sublitorânea. Entretanto, deve ser seguida a recomen- 
dação do procedimento CRIMP de que estratégias amostrais que produzem grandes volumes de amostras a serem triadas e identificadas devem ser usadas apenas em situações específicas, como quando há grande probabilidade de ocorrência de espécies introduzidas em um ambiente determinado.

A região entre marés, em especial, pode representar ambiente favorável ao desenvolvimento de determinadas espécies, particularmente resistentes à dessecação e a temperaturas extremas (Lüning 1990). Apesar de serem consideradas de menor prioridade, por não mostrarem fortes tendências a receber espécies introduzidas, coletas na região entre marés também são recomendadas pelo procedimento CRIMP, em casos específicos, sendo interessantes para fornecer a base para o estudo subseqüente da dispersão de espécies (Hewitt \& Martin 2001).

Recomenda-se que o levantamento florístico de áreas portuárias, como a baía de Sepetiba, e de outras áreas do litoral brasileiro reconhecidamente sujeitas à forte interferência do homem, seja feito de modo padronizado, consolidando o acompanhamento temporal das comunidades sob impacto, e viabilizando a consecução de medidas mitigadoras desses impactos.

$\mathrm{O}$ presente estudo pode servir como base para o detalhamento do Plano Nacional de Trabalho para a Gestão da Água de Lastro, a ser implementado pelo Ministério do Meio Ambiente, nos demais portos do Brasil, em conformidade com o Programa GloBallast.

\section{Agradecimentos}

À Organização Marítima Internacional, Programa de Desenvolvimento das Nações Unidas, e Fundo Mundial para o Meio Ambiente, pelo apoio financeiro ao projeto; à Mutue Toyota Fujii e Carlos Wallace do Nascimento Moura, pela confirmação da identificação de exemplares de Chondria e Laurencia e de Corallinaceae, respectivamente; à Maria Célia Villac e Alexandre de Carvalho Leal Neto, pela revisão do texto e todo o apoio durante a realização deste estudo; aos assessores científicos, pelas valiosas críticas e sugestões.

\section{Referências bibliográficas}

Amado Filho, G.M.; Barreto, M.B.B.; Marins, B.V.; Félix, C. \& Reis, R.P. 2003. Estrutura das comunidades fitobentônicas do infralitoral da Baía de Sepetiba, RJ, Brasil. Revista Brasileira de Botânica 26(3): 329-342.
Amado Filho, G. M. \& Marins, B. V. 2004. Fitobentos. Cap. 6, pp. 37-45. In: M.C. Villac; F.C. Fernandes; S. Jablonski; A.C. Leal Neto \& B.H. Coutinho (eds.). A biota da área sob influência do Porto de Sepetiba, Rio de Janeiro, Brasil: levantamento de dados pretéritos. Brasília, Ministério do Meio Ambiente.

Barreto, M.B.B. \& Yoneshigue-Valentin, Y. 2001. Aspectos morfológicos do gênero Ceramium (Ceramiaceae, Rhodophyta) no estado do Rio de Janeiro. Hoehnea 28(1): 77-110.

Bellorín, A. \& Oliveira, E.C. 2001. Introducción de especies exóticas de algas marinas: situación en América Latina. Pp. 693-701. In: K. Alveal \& T. Antezana (eds.). Sustentabilidad de la biodiversidad: un problema actual - bases científico-técnicas, teorizaciones y proyecciones. Concepción, Universidad de Concepción.

Berchez, F.A.S. \& Oliveira, E.C. 1992. Temporal changes in benthic marine flora of the Baía de Santos, SP, Brazil, over the last four decades. Pp. 120- 131. In: M. CordeiroMarino; M.T.P. Azevedo; C.L. Sant'Anna; N.Y. Tomita \& E.M. Plastino (eds.). Algae and environment: a general approach. São Paulo, Sociedade Brasileira de Ficologia e CETESB.

Boudouresque, C.-F. \& Verlaque, M. 2002. Biological pollution in the Mediterranean Sea: invasive versus introduced macrophytes. Marine Pollution Bulletin 44: 32-38.

Carlton, J.T. 1985. Transoceanic and inter-oceanic dispersal of coastal marine organisms: the biology of ballast water. Oceanography and Marine Biology, Annual Review 23: 313-371.

Carlton, J.T. 2001. Introduced species in U.S. coastal waters: environmental impacts and management priorities. Arlington, Pew Oceans Commission.

Carlton, J.T. \& Geller, J.B. 1993. Ecological roulette: the global transport of nonindigenous marine organisms. Science 261: 78-82.

Chamberlain, Y.M. \& Irvine L.M. 1994. Lithophylloideae. Pp. 58-112. In: L.M. Irvine \& Y.M. Chamberlain (eds.). Seaweeds of the British Isles. London, HMSO.

Cohen, A.N. \& Carlton, J.T. 1998. Accelerating invasion rate in a highly invaded estuary. Science 279: $555-558$.

De Wreede, R.E. 1985. Destructive (harvest) sampling. Pp. 147-160. In: M.M. Littler \& D.S. Littler (eds.). Ecological field methods: macroalgae. Cambridge, Cambridge University Press.

Figueiredo, M.A.O.; Barreto, M.B.B. \& Reis, R.P. 2004. Caracterização das macroalgas nas comunidades marinhas da Área de Proteção Ambiental de Cairuçú, Parati, RJ - subsídios para futuros monitoramentos. Revista Brasileira de Botânica 27(1): 11-17.

Grosholz, E.D.; Ruiz, G.M.; Dean, C.A.; Shirley, K.A.; Maron, J.L. \& Connors, P.G. 2000. The impacts of a nonindigenous marine predator in California bay. Ecology 81: 1206- 1224.

Guimarães, S.M.P.B.; Fujii, M.T.; Pupo, D. \& Yokoya, N.S. 2004. Reavaliação das características morfológicas e suas implicações taxonômicas no gênero Polysiphonia sensu lato (Ceramiales, Rhodophyta) do litoral dos estados de São Paulo e Espírito Santo, Brasil. Revista Brasileira de Botânica 27(1): 163-183. 
Hay, C.H. 1990. The dispersal of sporophytes of Undaria pinnatifida by coastal shipping in New Zealand, and implications of further dispersal of Undaria in France. British Phycological Journal 25: 301-313.

Hewitt, C.L. \& Martin, R.B. 2001. Revised protocols for baseline port surveys for introduced marine speciessurvey design, sampling protocols and specimen handling. Centre for Research on Introduced Marine Pests, Hobart. Technical Report n. 22, 46 p.

Horta, P.A. \& Oliveira, E.C. 2000. Morphology and reproduction of Anotrichium yagii (Ceramiales, Rhodophyta) - a new invader seaweed in the American Atlantic? Phycologia 39(5): 390-394.

Joly, A. B. 1965. Flora marinha bentônica do litoral norte do estado de São Paulo e regiões circunvizinhas. Boletim da Faculdade de Filosofia, Ciências e Letras da Universidade de São Paulo 294 (Bot. 21): 1-393.

Krebs, C.J. 1989. Ecological methodology. New York, Harper \& Row.

Leal Neto, A.C. \& Jablonski, S. 2004. O Programa GloBallast no Brasil. Pp. 11- 20. In: J.S.V. Silva \& R.C.C.L. Souza (orgs.). Água de lastro e bioinvasão. Rio de Janeiro, Editora Interciência.

Lüning, K. 1990. Seaweeds: their environment, biogeography, and ecophysiology. New York, John Wiley \& Sons.

Moura, C.W.N. \& Yamaguishi-Tomita, N. 1998. Jania ungulata f. brevior (Corallinales, Rhodophyta): nova ocorrência para o Atlântico. Hidrobiológica 8(2): $145-153$.

Oliveira, E.C. 1984. Algas exóticas nos mares brasileiros. Ciência e Cultura 36: 801-803.

Oliveira, E.C. \& Qi, Y. 2003. Decadal changes in a polluted bay as seen from its seaweed flora: the case of Santos Bay in Brazil. Ambio 32(6): 403-405.

Oliveira Filho, E.C. \& Berchez, F.A.S. 1978. Algas marinhas bentônicas da Baía de Santos - alterações da flora no período de 1957-1978. Boletim de Botânica da Universidade de São Paulo 6: 49-60.

Paula, E.J.; Pereira, R.T.L. \& Ostini, S. 1998. Introdução de espécies exóticas de Eucheuma e Kappaphycus (Gigartinales, Rhodophyta) para fins de maricultura no litoral brasileiro: abordagem teórica e experimental. v.2, Pp. 341-357. In: Anais do IV Congresso LatinoAmericano de Ficologia. Caxambu 1996. São Paulo, Sociedade Ficológica da América Latina e Caribe.

Paula, E.J. \& Oliveira, E.C. 2004. Macroalgas exóticas no Brasil com ênfase à introdução de espécies visando à maricultura. Pp. 99-112. In: J.S.V. Silva \& R.C.C.L. Souza (orgs.). Água de lastro e bioinvasão. Rio de Janeiro, Editora Interciência.
Pedrini, A.G.; Cassano V.; Coelho, L.G. \& Labronici, G.J. 1994. Macroalgas marinhas da região sob influência da Central Nuclear Almirante Álvaro Alberto, Angra dos Reis, RJ, Brasil. I - Composição taxonômica. Pp. 727-731. In: Anais do V Congresso Geral de Energia Nuclear. Rio de Janeiro 1994. Associação Brasileira de Energia Nuclear. v.2.

Pinheiro-Vieira, F. \& Ferreira, M.M. 1968. Segunda contribuição ao inventário das algas marinhas bentônicas do nordeste brasileiro. Arquivos da Estação de Biologia Marinha da Universidade Federal do Ceará 8: 75-82.

Reis, R.P. \& Yoneshigue-Valentin, Y. 1998. Variação espaçotemporal de populações de Hypnea musciformis (Rhodophyta, Gigartinales) na Baía de Sepetiba e Armação de Búzios, RJ, Brasil. Acta Botanica Brasilica 12(3): 465-483.

Ruiz, G.M.; Fofonoff, P.W.; Carlton, J.T.; Wonham, M.J. \& Hines, A.H. 2000. Invasion of coastal marine communities in North America: apparent patterns, processes, and biases. Annual Review of Ecology and Systematics 31: 481-531.

Silva, P.C.; Basson, P.W. \& Moe, R.L. 1996. Catalogue of the benthic marine algae of the Indian Ocean Berkeley, University of California Press.

Smith, J.E.; Hunter, C.L. \& Smith, C.M. 2002. Distribution and reproductive characteristics of non-indigenous and invasive marine algae in the Hawaiian Islands. Pacific Science 56(3): 299-315.

Széchy, M.T.M. \& Paula, E.J. 2000. Macroalgas associadas a bancos de Sargassum C. Agardh (Phaeophyta, Fucales) do litoral dos estados do Rio de Janeiro e São Paulo, Brasil. Hoehnea 27(3): 235-257.

Ugadim, Y. 1973. Algas marinhas bentônicas do litoral sul do estado de São Paulo e do litoral do estado do Paraná. III - Divisão Rhodophyta, 3 - Ceramium (Ceramiaceae, Ceramiales). Boletim de Zoologia e Biologia Marinha, N.S. 30: 691-7812.

Underwood, A.J. 1997. Experiments in Ecology: their logical design and interpretation using analysis of variance. Cambridge, Cambridge University Press.

Verlaque, M. 1994. Inventaire des plantes introduites en Méditerranée: origines et répercussions sur l' environnement et les activités humaines. Oceanologica Acta 17(1): 1- 23.

Villalard-Bohnsack, M. \& Harlin, M.M. 1997. The appearance of Grateloupia doryphora (Halymeniaceae, Rhodophyta) on the northeast coast of North America. Phycologia 36: 324-328.

Warwick, R.M. 1993. Environmental impact studies on marine communities - pragmatical considerations. Australian Journal of Ecology 172: 215-226.

Wynne, M.J. 1998. A checklist of benthic marine algae of the tropical and subtropical western Atlantic: first revision. Nova Hedwigia 116: 1-155. 